\title{
Improved Leach using Highest Remaining Energy First based Cluster Head Selection
}

\author{
Navjot Kaur \\ Department of Computer Science \& Engineering, \\ Sri Sai College of Engineering \& Technology, \\ Manawala, Amritsar
}

\author{
Gagandeep Singh \\ Department of Computer Science \& Engineering, \\ Sri Sai College of Engineering \& Technology, \\ Manawala, Amritsar
}

\begin{abstract}
Power effectiveness found to be main limitation in WSN due to insufficient battery of sensor nodes. Therefore, the most significant focus of the existing work is to locate the ways to decrease the difficulty of power utilization and will result in improvement in the network stability period and life time. A lot of researchers have planned a variety of the protocols to additional improve the network lifetime. This paper has evaluated the issues which have been ignored in the field of the WSNs. In this paper, a latest enhanced highest residual power initial based clustering technique is planned to improve the results of the existing iLEACH protocol. Proposed technique is an extension to iLEACH using sorting techniques to improve the results additional. The proposed algorithm is designed and implemented in MATLAB. It has been exposed in this paper that the proposed algorithm provides improved results than existing clustering protocols.
\end{abstract}

Keywords: WSN, LEACH, Energy Efficient, Sorting Energy.

\section{INTRODUCTION}

A Wireless Sensor Networks (WSN) consists of spatially dispersed independent sensors node to cooperatively monitor physical or environmental conditions, such as temperature, sound, vibration, pressure, motion or pollutants. WSN contains a large number of sensor nodes with a limited power supply. A sensor network consists of sensor nodes that can communicate with each other through wireless links. Sensors nodes are be remotely deployed in large numbers and operates separately in unattended surroundings. In wireless sensor network, there are lots of challenges. Typically a sensor network consists of a fusion middle and a large number of in homogeneously placed sensor nodes. The goal of the sensor nodes is to cooperatively monitor some physical or environmental quantity. Each sensor node in the wireless sensor network takes a sound corrupted dimension of the unknown quantity; these dimensions are then sent to the fusion centre where an estimation is produced. A major challenge for these sensor nodes is that they have limited and non-renewable power sources. Thus power effectiveness is a major concern, since it allows us to maximize the lifetime of the wireless sensor network. Wireless sensor Network lifetime has become the key characteristic for evaluating wireless sensor networks in an application-specific way. Particularly the availability of sensor nodes, the sensor coverage, and the connectivity have been included in discussions on network lifetime. Even quality of service measures can be reduced to lifetime considerations. A great number of algorithms and methods were planned to raise the lifetime of a wireless sensor network - while their evaluations were always based on a particular definition of sensor nodes network lifetime.
There are several definitions of the sensor nodes network lifetime. Lifetime can be defined as the time until the initial sensor nodes runs out of power .Others have used definitions that include fractions of surviving sensor nodes. The lifetime is the periods in which the network can perform its desired job with acceptable quality. The max-min approach is used to exploit the state information of the network to maximize the minimum remaining power across the network. Most of the past work in this area focus on system-wide power effectiveness.

\section{LITERATURE SURVEY}

Heinzelman et al. (2000) [1] has planned the first Leach protocol ever. Wireless network dispersed micro sensor systems that provide the reliable observing the areas for military and civil applications have been explained. It has also explained that the communication protocols, which have complete the capable improvement on the overall power dissipation of WSN. Direct communication, multi hop routing, and static clustering have been considerate additional capable in wireless sensor networks, So LEACH (LowEnergy Adaptive Clustering Hierarchy), a clustering-based protocol has been planned that have developed non randomized proposal for cluster-heads. Localize association has exploited scalability and toughness in the wireless sensor networks, and data mixture has reduced the amount of data to the base station. A high amount of energy reduction has been achieved as compared with conservative routing protocols. Guisheng Yin et al. [2008] [2] Since the sensor nodes of networks are in the situation of a highly-limited and un replenishable power resource such as battery energy, calculation, and storage space, the power effectiveness is the most significant key point of the wireless sensor network routing designing. In this paper, A novel routing algorithm which combines with hierarchical routing and geographical routing is planned. Based on the hierarchical wireless sensor network architecture, the procedure of forwarding packets between the supply nodes in the target area and the sink consists of two phases - inter cluster routing and intra-cluster routing, a greedy algorithm is adopted in the procedure of the inter-cluster routing and an multi-hop routing algorithm based on the forwarding limit angle is designed for the intra cluster routing. The analysis and simulation results show that our routing algorithm has improved performance in conditions of power utilization and delay, it is appropriate for the information transmission in a high-density network. Sun et al. (2009)[3] has planned a method in which some process has done to basic LEACH, named as ILEACH.ILEACH has based on the feature of limited energy of sensor networks to extend the life time of the total networks. Consideration of Wireless sensor Nodes for cluster head option has been done on the basis of remaining energy. The limit threshold of space 
has used to optimize cluster scheme. Building of the routing tree has been designed on the basis of Cluster heads' weight. A tree based routing has been done in which a cluster head is selected as root sensor node and the criterion for selecting root sensor node is to be closer to the base station and owing sufficient power. A improved presentation in both network life period and cluster head selection has been print out as a conclusion. Babied et al. (2010)[4] have proposed a book technique to select a cluster Head. LEACH protocol has put threshold value to 0 for next $1 / \mathrm{p}$ rounds when a sensor node has been elected as a cluster head. This method optimized LEACH technique, by adjusting threshold allowing for a few factors. Proposed Algorithm has recognized the threshold of each sensor node respectively to the number of live and dead sensor nodes in every round, so the probability for additional sensor nodes has been established to become cluster head. Energy issue has taken into consideration in this method, at some stage in Cluster Head election phase and no-clusterhead electing sensor node as its cluster head, while data transmits procedure is the same as LEACH. This algorithm considered the number of live and life less sensor nodes in each round to estimate the threshold value. Probability of choose the cluster-head has been increasing after rounds. Consideration of number of live and life less sensor nodes in each round has been to calculate the Threshold. It finished that the proposed method can reduce the low energy stage sensor nodes to be elected as cluster heads, and set up the energy stability of set of connections load. Additionally, Results have been achieved improved network lifetime in WSN. Therefore, the technique to modify the threshold may be an well-organized technique to find out the problem of network energy consumption as this method explained. Bark and Lillian (2010) [5] have prepared focus mostly on extending the WSN lifetime. Lifetime has been extended by creation WSNs redundant by adding up further sensor nodes. The passive (switched off) spares has been made available to become active (be switched on) whenever any active WSN sensor node energy exhausted. A latest planned LEACH-SM (LEACH Spare Management) has modified the famous LEACH protocol by enhancing it with an well-organized association of spares. Addition of the spare option stage has been finished in LEACH; this functionality has been named as further administration features in LEACH-SM. During additional option stage, each sensor node has been decided in parallel whether it would be become an active primary sensor node, or a passive extra sensor node. The sensor nodes decided spares go asleep, while the WSN as the whole has been maintained the necessary above-threshold target reporting. (The spares have awakened when the probability that any primary sensor node exhausted its power reaches a undefined value.) Identification of spares alone has been increased energy efficiency for WSN as proved, Decentralized energy-efficient further election method has been used in spare election stage by extra manger. Reduce in the time of the active time for cluster heads has been observed, considered as a side effect. Decreased energy consumption by cluster heads has been observed mainly. Linlin Wang et al. [2010] [6] WSN is composed of hundreds of sensor nodes involving in limited power, well-organized and low-power consuming routing algorithm is the critical trouble to the routing design. In this paper, the LEACH protocol, which is a classic clustering routing protocol, has been researched and improved based on energy, the new algorithm is different from the LEACH in the technique of determining the number of clusters heads and the criterion that chooses cluster-heads. Simulation results point to that the new algorithm has the advantages of reducing power consuming and prolonging the lifetime of the wireless sensor network. Melese and Gao (2010) [7] have explained the energy consumption of sensor nodes in WSN. Main effort has been complete for balancing the energy consumption across the wireless sensor network so that continued existence time of all sensor nodes can increase. Optimization of the energy consumption has been focused by taking consumed energy as a main factor for criteria cluster head election. Energy consumption factor have contributed extra capably in increase network life time of WSN rather than residual energy. By considering energy consumption, new formula has been planned to compute threshold value. In order to optimize energy consumption and increase network life time, it is essential to balance energy between sensor nodes has been summarized. Extension in the LEACH formula has been complete on the basis of a part that includes the consumed energy of each sensor nodes of WSN, A increase in network life time has been observed. Main impact has been seen in the cases when long distances occurred between the base station and the sensor nodes. Remarkable development has been concluded for cluster head election. Tao et al. (2010) [8] have represented a ladder based protocol in WSN. A new energyefficient protocol has been discussed by employing the cluster member energy threshold problem to limit the configuration of very big and the small clusters at the same period. Conceptualization of energy rating technique has been complete to stay away from uneven energy allocation; calculated energy has been also taken into description to decrease theenergyr utilization of cluster members. An enhancement has been complete by taking the consideration of three factors: unstable Clusters, irregular energy allocation and unnecessary energy utilization when Cluster Head is expired. Hierarchical or multi-hop routing approach has been used to obtain data from the cluster head sensor nodes to the base station. Cluster heads have planned such a mode that they form a multi-hop backbone for communicating information among cluster heads until they reach the base station. An Algorithm has been done considerably increases the network life period. Peng et al. (2011) [9] have proposed a current method in which adaptive clustering hierarchy algorithm has been proposed to meet QOS (Quality of Services) needs. Modification has been complete in basic LEACH and an improved protocol has elaborated in which improvement has been occurred in the energy efficiency and other QOS parameters by excluding the sensor node with improper geographic position to be the cluster heads. The optimal measuring range of head sensor nodes has been planned to be a criterion of cluster head election, and each cluster heads has been selected according to the sensor node thickness threshold, which is defined by the sensor node allocation state process and transmission between sensor nodes. A development has been shown in the network life period and the communication value by electing the Cluster head in the region of proper sensor node mass. To get outstanding results when there is irregular allocation of sensor nodes. Chen and Wang (2012) [10] have explained an improved model in Wireless sensor network which has been based on heterogeneous energy of sensor nodes for similar first energy and multiple hop data communication between cluster heads is proposed. New threshold has been introduced on the base of current energy and average energy of the sensor node to cluster head election probability and provide reliability that higher residual energy have greater probability to become cluster heads than that with the low residual energy. Problem of number of cluster heads reduces with the increase of the number of rounds. Confirmation has been provided with the approach that sensor nodes with higher 
residual energy have greater probability to become cluster heads than that with the low residual energy. Extension in the network lifetime and guarantees a well spread energy consumption model been established. Meenakshi Sharma et al. [2012] [11] Routing protocols like EEE LEACH, LEACH and Direct communication protocol (DTx) in Wireless Sensor Network (WSN) and a comparison study of these protocols based on some performance matrices. Addition to this an attempt is complete to compute their communication time and throughput. To compute these, MATLAB environment is used. Finally, on the basis of the obtained outcome from the simulation, the above mentioned three protocols are compared. The comparison outcome show that, the EEE LEACH routing protocol has a greater communication time than LEACH and DTx protocol and with smaller throughput. Thu Ngo Quynhet al. [2012] [12] Wireless Sensor Network (WSN) is a promising approach for a variety of applications. Because of limitation of power resource, memory space and processing ability of sensor nodes, it is very difficult to implement IP-based routing protocols in WSN. Recently, many research focus on developing particular routing protocols for WSNs with the main design criteria: power effectiveness, load balance and reliability. The most well known routing protocol class is hierarchical which divides wireless sensor network into many clusters. Each cluster is represented by a cluster head $(\mathrm{CH})$ that is responsible for receiving information from all non-CH members, aggregating these information and sending to the sink. In order to balance power utilization of the entire wireless sensor network, $\mathrm{CHs}$ are not fixed but rotated. Therefore, the hierarchical protocols can decrease and balance the power utilization and extend network lifetime. LEACH is one of the initial cluster-based for wireless sensor network, which includes distributed cluster formation. In this paper, we propose a new hierarchical routing protocol (called EL-LEACH: Energy and Load balance LEACH) which achieves power effectiveness and load balance. Our simulation outcome shows that our new scheme EL-LEACH achieves better power utilization, load balance and network life time than other well known LEACH protocols. Beiranavand et al. (2013) [13] have planned a improvement in LEACH named I-LEACH, An enhancement has been done by considering mainly three factors; residual energy in sensor nodes, Distance from base station and number of neighbouring sensor nodes. Sensor node has been considered as head sensor node if it has optimal value for discussed three factors i.e. have more residual energy as compare to average power of WSN, more neighbours than average neighbours for a sensor node calculate in WSN and sensor node having less distance from base station as comparison to sensor node's average distance from base station in wireless sensor network. Decrease in energy utilization and maintenance in wireless sensor network life time has been observed. Sipra Das Bit; et al. (2013) [14] using "homogeneous sensor nodes" in a paper entitled "Improvement of WSN lifetime by deploying heterogeneous nodes" investigated the difficulty for enhancing wireless sensor network lifetime using homogeneous sensor nodes. It was revealed that power imbalance in Wireless sensor network occurs due to relaying of information from different parts of the wireless sensor network towards base station. So for better power balance instead confusing only sensor nodes it is desirable to organize relay sensor nodes in addition to sensor nodes to manage such imbalance. Allocation-wise predetermined heterogeneous sensor node deployment strategy based on the principle of power balancing derived from this analysis, leading to an improvement of wireless sensor network lifetime was developed. Exhaustive simulation is performed primarily to metrics. Halfwit and Malice (2014) [2] has proposed a latest method in which theory of Vice Cluster head has been taken out. Vice Cluster head has been selected as alternating cluster head that has worked when the cluster head has fall downwards. Criteria for the election of vice cluster head have situate on the basis of three factors i.e. Minimum distance, maximum residual energy, and minimum energy. Development in the wireless sensor network life has been obtained because of the cluster head has not dead forever. As a cluster head has been died it has been replaced by its vice Cluster head.

\section{PROPOSED WORK}

Due to limited battery in sensor nodes, energy is main constraint in wireless sensor network. So focus of present work will be minimizing the energy consumption and enhancement in the wireless sensor network stability period and life span.

Step 1: Initial of all initialization of the wireless sensor network will be complete by setting up a variety of constants and variables of the wireless sonsor network. Like diameters of wireless sensor network, distance of sink from the wireless sensor network, no of nodes, probability of a node to become cluster head, power supplied to each sensor node, transmitter power per sensor node, receiver power per mode, amplification power, distance between cluster head and sink etc. However as this research work focuses on the mobile base station therefore the location of the base station remains moveable but it is supposed that it will stay within the sensor field.

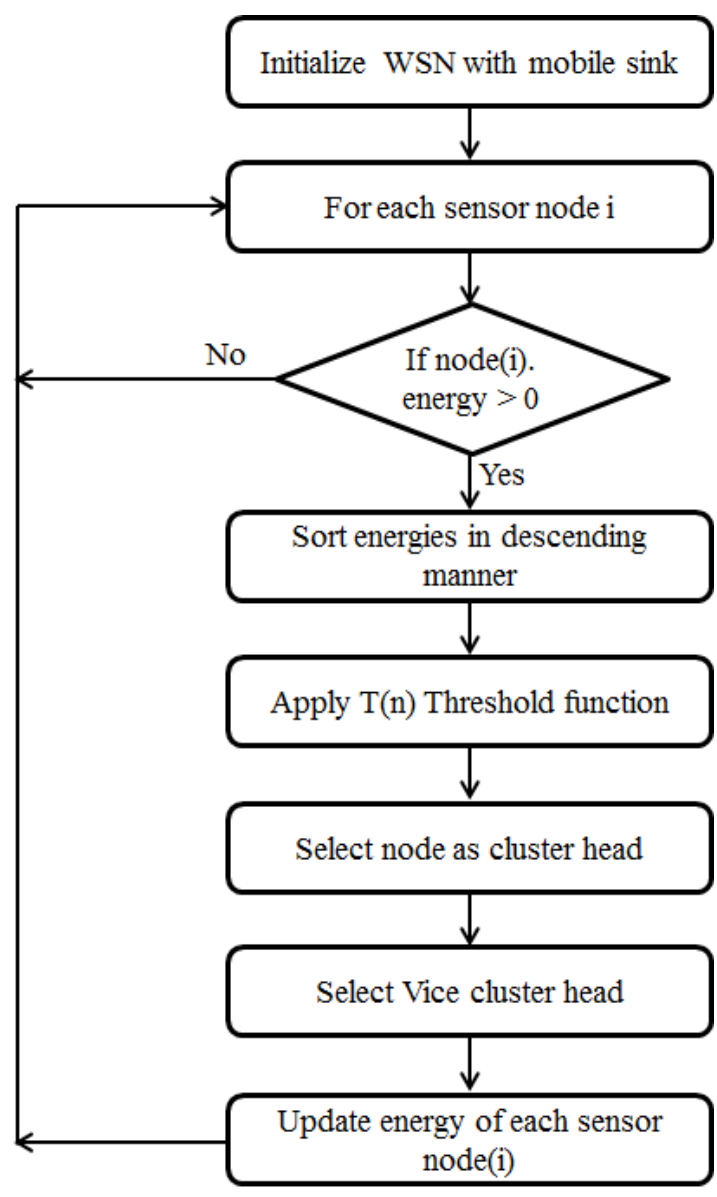

Fig 1: Flow Chart of Proposed Solution 
Step2: For all sensor node i if any node has 0 or negative power then sensor node will be set as lifeless and continue to next step.

Step3: If node(i) energy>avg remaining power then move to step 4 else moves to step 2.

Step4: Now sensor node sorting will be complete to find the sensor node with highest power.

Step 5: Now select sensor node as cluster head based upon $\mathrm{T}(\mathrm{n})$ Thresholding function,

$$
=\left\{\begin{array}{c}
\frac{p}{1-p\left(\operatorname{rmod}\left(\frac{1}{p}\right)\right.} * \frac{E_{c u r}}{E_{\text {avg }}} * \frac{N b r_{n}}{N b r_{\text {avg }}} * \frac{d t o B S_{\text {avg }}}{d t o B S_{n}} \quad S \in G \\
\text { Otherwise }
\end{array}\right.
$$

Step 6: Now Tx and Rx operations come in action for sending and receiving the packets.

$$
D=\sqrt{\left(x d_{1}-x d_{2}\right)^{2}+\left(y d_{1}-y d_{2}\right)^{2}}
$$

Step 7: Evaluate power dissipated using predefined equations. Evaluate the power dissipation and update the residual energies it. Where distance will be evaluated using eq. 5 [2] and updating of power will be based upon the eq. 6 [13] and eq. 7 [13].

$$
\begin{aligned}
\mathrm{d}_{\mathrm{toCH}} & =\frac{\mathrm{M}}{\sqrt{2 \pi \mathrm{k}}}, \mathrm{d}_{\text {toBS }}=0.765 \frac{\mathrm{M}}{2} \\
\mathrm{E}_{\mathrm{Tx}}(\mathrm{l}, \mathrm{d}) & =1 \mathrm{E}_{\text {elec }}+l \varepsilon_{\mathrm{fs}} d^{2}, \mathrm{~d}<d_{0} \\
\mathrm{E}_{\mathrm{Tx}}(\mathrm{l}, \mathrm{d}) & =1 \mathrm{E}_{\text {elec }}+l \varepsilon_{\mathrm{mp}} d^{4}, \mathrm{~d} \geq d_{0}
\end{aligned}
$$

Step 8: Update residual power of each sensor node (i) and move to step 2 again.

\section{RESULTS AND DISCUSSIONS}

Once the implementation starts, the initial view that comes to be perceived is shown in Figure. The screen is divided in to a variety of regions that are called clusters. Each cluster thus formed has a cluster head, normal, advance sensor node and super sensor nodes. The entire network has a sink that is responsible for the collection of information from all other sensor nodes.

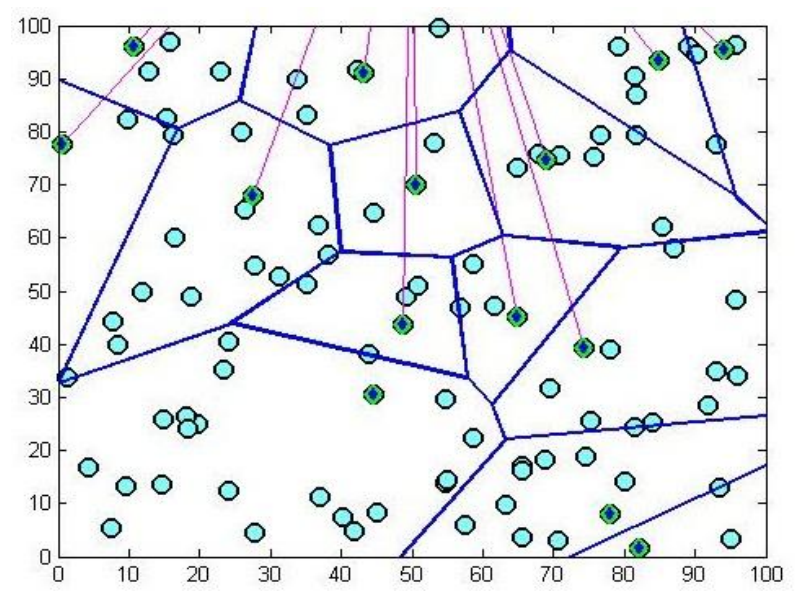

Fig 2: when all the sensor nodes are active
Figure 2 has shown the active environment of LEACH protocol. Green circled sensor nodes are representing normal sensor nodes whereas sensor nodes with diamonds are representing cluster heads. The blue lines, forming hexagonal shape are cluster head region also called cluster field. Each cluster field has only one cluster head. Magenta lines are representing the communication of cluster head with the sink. The sink is residing outside the wireless sensor network field.

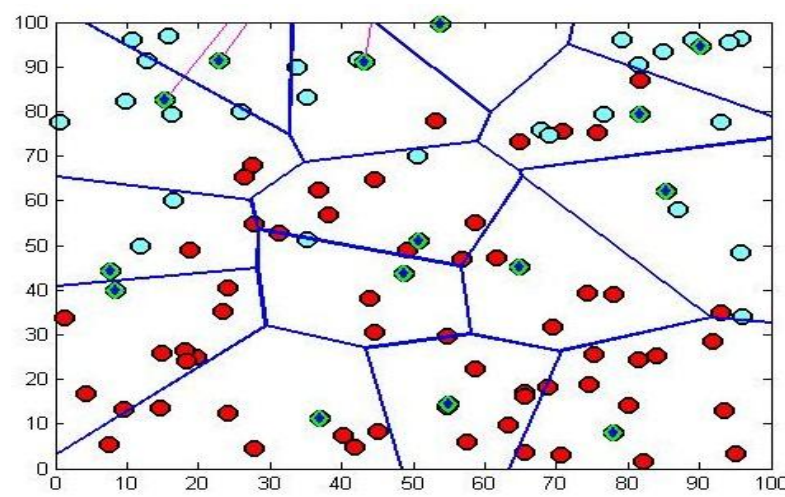

Fig 3 when some of the sensor nodes are dead

Figure 3 has shown the active environment of LEACH protocol. Green circled sensor nodes are representing normal sensor nodes whereas sensor nodes with diamonds are representing cluster heads. The blue lines, forming hexagonal shape are cluster head region also called cluster field. Each cluster field has only one cluster head magenta lines are representing the communication of cluster head with the sink. The sink is residing outside the sensor field. circle with red colors are representing the normal sensor nodes that are lifeless. A sensor node is known as lifeless if it has zero power that is it is no longer available for communication.

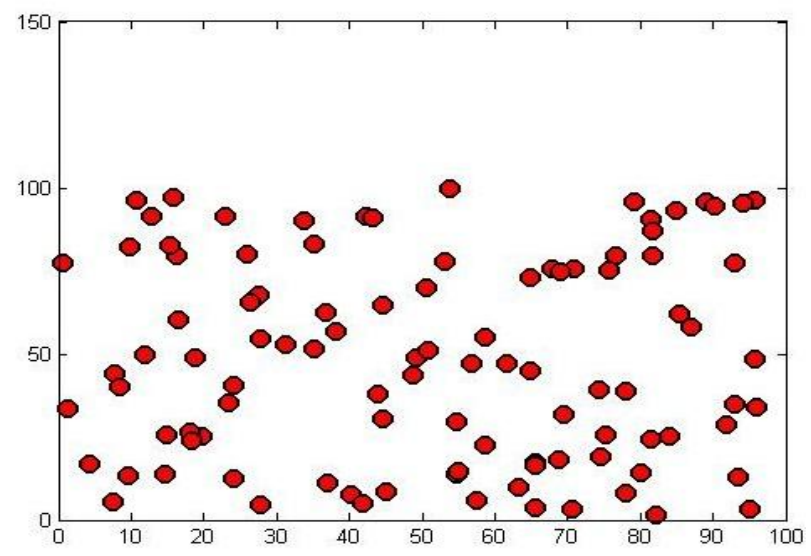

Fig 4: When all the sensor nodes are dead

Figure 4 is showing the end of the sensor environment of sensor network where all the sensor nodes are lifeless. The small red diamond is representing sensor nodes that are lifeless. A sensor node is known as lifeless if it has zero power that is it is no longer available for communication. 


\section{PERFORMANCE EVALUATION}

This section contains the comparison among the LEACH, iLEACH and the planned method.

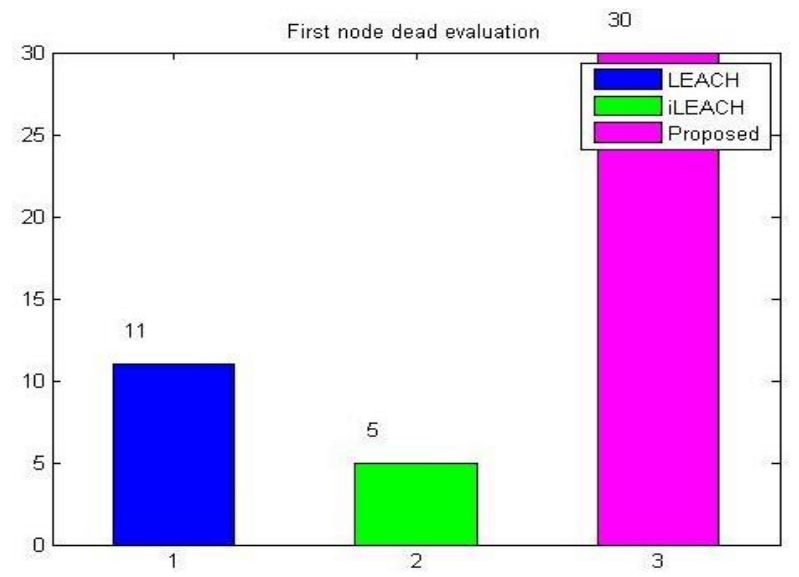

Fig 5: WSNs with first node dead time evaluation

Figure 5 has shown the histogram of the initial sensor node lifeless time. It is clearly shown that the initial sensor node lifeless has been prolonged than the LEACH and ILEACH. Therefore planned algorithm has important enhancement over the LEACH and ILEACH.

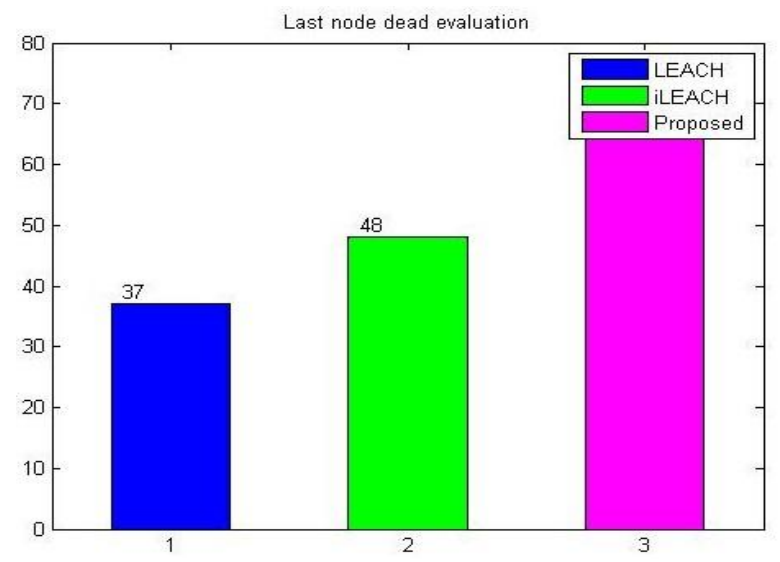

Fig 6: WSNs with Last node dead time evaluation.

Figure 6 has shown the histogram of the last sensor node lifeless time. Last sensor node lifeless time is also called the wireless sensor network lifetime. It is evidently publicized that the last sensor node lifeless time has been increased than the LEACH and ILEACH. Therefore projected algorithm has important improvement over the LEACH and ILEACH.

Figure 7 has shown the comparison among LEACH, ILEACH and planned with respect to average consumed power. It has been clearly shown that the average consumed power with respect to rounds in case of the planned is quite less than the LEACH and ILEACH. It has evidently proved that the planned algorithm is quite improved than the available techniques.

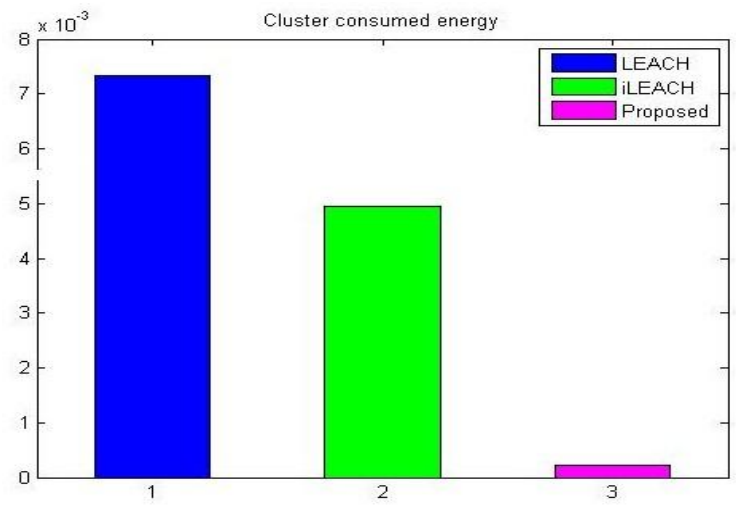

Fig 7: Average consumed energy in rounds evaluation

\section{CONCLUSION}

The incomplete battery of sensor nodes is found to be serious problem. Thus effectiveness of power becomes major constraint in wireless sensor network. This research work has focused to locate the ways to reduce the problem of the power utilization. This paper evaluated the issues which have been ignored in the field of the WSNs. This paper has planned a novel enhanced highest residual power initial based clustering method to get better the outcome of the existing iLEACH protocol additional. Planned method is an addition to iLEACH using sorting techniques to get better the outcome additional. Planned algorithm is designed and implemented in MATLAB. It has been shown that the planned algorithm provides outcome improved than existing clustering protocols.

The planned algorithm is designed and implemented in MATLAB. The outcome have shown considerable enhancement over the available techniques. In nearby future, we will use fuzzy based cluster head election to get better the cluster head selection additional.

\section{REFERENCES}

[1] Zhi-fengDuan, Fan Guo, Ming-xing Deng and Min Yu "Shortest Path Routing Protocol for Multi-layer Mobile Wireless Sensor Networks", International Conference on Net works Security, Wireless Communications and Trusted Computing (IEEE) (2009), 25-26 April; Wuhan, Huber, pp.106-110.

[2] Heinzelman, W., Chandrakasan, A., and Bala krishnan, H., "Energy-Efficient Communication Protocol for Wireless Microsensor Networks" IEEE Proceedings of the 33rd Hawaii International Conference on System Sciences, Jan 2000, pp. 3005-3014.

[3] Liu, Y., Luo, Z., Xu, K. and Chen, L., "A Reliable Clustering Algorithm base on LEACH Protocol in Wireless Mobile Sensor Networks" International Conference on Mechanical and Electrical Technology, September 2010, pp. 692-696.

[4] Ahlawat, A. and Malik, V., "An Extended Vice-Cluster Selection Approach to Improve V-LEACH Protocol in WSN", IEEE $3^{\text {rd }}$ conference on Advance Computing and Communication Technology, April 2013, pp. 236-240. 
[5] Guisheng Yin1, Guang Yang1,2, Wu Yang1,2, Bingyang Zhang1,2, Wenjin Jin1,2 "An Energy-Efficient Routing Algorithm for Wireless Sensor Networks", International Conference on Internet Computing in Science and Engineering (IEEE) (2008),28-29 Jan; Harbin, pp.181-186.

[6] Yuhua Liu Yongfeng Zhao JingjuGao "A New Clustering Mechanism Based On LEACH Protocol", International Joint Conference on Artificial Intelligence (IEEE) (2009), 25-26 April; Hainan, Island, pp.715-718.

[7] Ma Chaw Mon Thein, Thandar Thein "An Energy Efficient Cluster-Head Selection for Wireless Sensor Networks", International Conference on Intelligent Systems, Modelling and Simulation (IEEE) (2010), 2729 Jan ; Liverpool, pp.287-291.

[8] Melese, D. G., Xiong, H., and Gao Q., "Consumed Energy as a Factor For Cluster Head Selection in Wireless Sensor Networks", IEEE 6th International Conference on Wireless Communications Networking and Mobile Computing (WiCOM), September 2010, pp. $1-4$

[9] Katiyar, V., Chand, N., Gautam, G. C. and Kumar A., "Improvement in LEACH Protocol for Large-scale Wireless Sensor Networks", IEEE Conference on Emerging Trends in Electrical and Computer Technology (ICETECT), March 2011, pp. 1070-1075.
[10] Sun, J., Chen, W., Zhang, B., Liu, X. and GU, X., "Energy-efficient Clustering Routing Protocol Based on Weight", IEEE International Conference on Wireless Communications \& Signal Processing, November 2009, pp. $1-5$

[11] Babaie, S., Agaalizadeh, S. and Golsorkhtabar, M. "The Novel Threshold Based Hierarchical Clustering Method for Wireless Sensor Network", IEEE International Conference on Electronics and Information Engineering (ICEIE), August 2010, pp. $191-195$

[12] Melese, D. G., Xiong, H., and Gao Q., "Consumed Energy as a Factor For Cluster Head Selection in Wireless Sensor Networks", IEEE 6th International Conference onWireless Communications Networking and Mobile Computing (WiCOM), September 2010, pp. $1-4$.

[13] Hongjoong Sin, Jangsoo Lee, Sungju Lee, SeunghwanYoo, Sanghyuck Lee, Jaesik Lee, YongjunLee, and Sungchun Kim, "Agent-based Framework for Energy Efficiency in Wireless Sensor Networks", World Academy of Science, Engineering and Technology 22, 2008, pp.305-309.

[14] Sun, J., Chen, W., Zhang, B., Liu, X. and GU, X., "Energy-efficient Clustering Routing Protocol Based on Weight", IEEE International Conference on Wireless Communications \& Signal Processing, November 2009, pp. 1-5. 